\title{
Unified Bit-Based Probabilistic Data Association Aided MIMO Detection for High-Order QAM
}

\author{
Shaoshi Yang ${ }^{1}$, Tiejun $\mathrm{Lv}^{2}$ and Lajos Hanzo ${ }^{1}$ \\ ${ }^{1}$ School of Electronics and Computer Science, University of Southampton, SO17 1BJ, UK \\ Email: $\{$ sy7g09, lh $\} @$ ecs.soton.ac.uk, http://www-mobile.ecs.soton.ac.uk \\ ${ }^{2}$ School of Information and Communication Engineering, Beijing University of Posts and Telecommunications, Beijing, 100876, China
}

\begin{abstract}
A unified Bit-based Probabilistic Data Association (B-PDA) detection approach is proposed for Multiple-Input Multiple-Output (MIMO) systems employing high-order Quadrature Amplitude Modulation (QAM). The new approach transforms the symbol detection process of QAM to a bit-based process by introducing a Unified Matrix Representation (UMR) of QAM. Both linear natural and nonlinear Gray bit-to-symbol mapping schemes are considered. Our analytical and simulation results demonstrate that the linear natural mapping based B-PDA approach attains an improved detection performance, despite dramatically reducing the computational complexity in contrast to the conventional symbol-based PDA aided MIMO detector. Furthermore, it is shown that the linear natural mapping based B-PDA method is capable of approaching the lower bound performance provided by the nonlinear Gray mapping based BPDA MIMO detector. Since the linear natural mapping based scheme is simpler and more applicable in practice than its nonlinear Gray mapping based counterpart, we conclude that in the context of the uncoded B-PDA MIMO detector it is preferable to use the linear natural bit-to-symbol mapping, rather than the nonlinear Gray mapping.
\end{abstract}

Index Terms-probabilistic data association, unified matrix representation, high-order QAM, low complexity detection.

\section{INTRODUCTION}

$\mathbf{T}$ HE Probabilistic Data Association (PDA) method, which was originally conceived for target tracking [1], may also constitute a promising detection technique. Firstly, it may achieve a near-optimal detection performance, especially in the context of Code Division Multiple Access (CDMA) systems [2][3]. Secondly, it has a low complexity which, increases no faster than $O\left(L^{3}\right)$, where $L$ is either the number of users in CDMA [2] or the number of transmit antennas in the MIMO systems [4]. Furthermore, the higher the number of transmit antennas or users, the better its performance, provided that the system is not rank-deficient [5]. Although the PDA approach strikes an excellent tradeoff between the attainable performance and the complexity imposed, it may be readily shown that the performance versus complexity benefits of the conventional symbol-based PDA-aided MIMO detectors in [4]-[6] are not as convincing as those of their CDMA-based counterpart [2]. According to the results of [5], one of the reasons is that the number of transmit antennas in a MIMO system is typically lower than that of the users in CDMA. A second reason is that typically QAM is used in MIMO systems, rather than BPSK. Furthermore, the equivalent "channel" matrix of CDMA systems is more likely to be well-conditioned than that

The financial support of the National Natural Science Foundation of China (NSFC) under Grant NSFC-60972075, the China Scholarship Council (CSC), as well as that of the EPSRC, UK and of the EU under the auspices of the OPTIMIX project is gratefully acknowledged. of MIMO systems ${ }^{1}$. As argued in [2], a key feature of PDA is the repeated conversion of a multimodal Gaussian mixture probability to a single Gaussian distribution having a matched mean and covariance. Hence the accuracy of the Gaussian approximation dominates the attainable performance. In fact, it has been demonstrated in [5] that the quality of the Gaussian approximation in PDA is the best for a large number of transmit antennas and a small number of modulation constellation points. Therefore, the performance of the conventional symbol-based PDA MIMO detectors degrades significantly for high-order QAM compared to the Maximum Likelihood (ML) detector, while its complexity increases substantially owing to the increased number of symbol probabilities to be computed.

Against this background, we aim to develop an efficient PDA-based MIMO detector for high-order QAM constellations. The novel contributions in this paper are as follows.

1) We present an explicit Unified Matrix Representation (UMR) of both linear natural and nonlinear binary reflected Gray mappings based rectangular QAM employed in MIMO systems, including BPSK modulation as a special case.

2) Based on the UMR, we propose a Bit-based PDA (B-PDA) MIMO detector for high-order rectangular QAM. The B-PDA transforms the conventional symbol-based QAM detection of MIMO systems [4]-[6] to a BPSK-like binary scenario, thus eliminating symbol-based decisions, and directly operates at the bit-level. While a similar mathematical representation of the linear natural mapping based rectangular QAM was also used in the context of the SDR technique of [7] and in the multi-level bit-interleaved coded modulation scheme of [8], its extension to the nonlinear binary reflected Gray mapping and its application to improving the PDA-based MIMO detectors have not been suggested before.

3) We investigate the complexity of the proposed B-PDA MIMO detector both analytically and by simulations. It is demonstrated that the linear natural mapping based B-PDA substantially reduces the computational complexity compared to the conventional symbol-based PDA MIMO detector in uncoded VBLAST systems using high-order QAM.

4) In addition to a remarkable complexity reduction, the simulation results shows that the linear natural mapping based B-PDA has an improved performance in comparison to the conventional symbol-based PDA. Furthermore, it approaches the lower bound performance provided by the binary reflected Gray mapping based B-PDA under the idealized perfect modulation matrix estimation assumption.

\footnotetext{
${ }^{1}$ The equivalent "channel" matrix in CDMA systems is usually the crosscorrelation matrix of the well-designed spreading codes. Typically it is wellconditioned. However, in MIMO systems it is possible that the channel matrix becomes rather ill-conditioned for some realizations. According to the Central Limit Theorem, the fewer independent random variables imposed by the illconditioned matrices may result in poor Gaussian approximations
} 
5) Considering the additional complexity and the potential performance degradation entailed by the modulation matrix estimation for binary reflected Gray mapping based B-PDA, we argue that it is preferable to use the simpler and more practicable linear natural mapping for the B-PDA detector in the context of the uncoded VBLAST system considered.

\section{Problem Statement}

Consider a spatial multiplexing MIMO system using $N_{T}$ transmit and $N_{R}$ receive antennas. The received baseband signal at each symbol instant is given by

$$
\mathbf{y}=\mathbf{H s}+\mathbf{n},
$$

where $\mathbf{H}$ is the $\left(N_{R} \times N_{T}\right)$-element complex channel matrix, $\mathbf{s}$ is the length- $N_{T}$ vector of transmitted symbols from a modulation constellation $\mathcal{A}=\left\{a_{1}, a_{2}, \cdots, a_{M}\right\}$, and $\mathbf{n}$ represents the length- $N_{R}$ complex-valued circularly symmetric Gaussian noise vector with a zero mean and a covariance matrix of $N_{0} \mathbf{I}$, where $\mathbf{I}$ is an $\left(N_{R} \times N_{R}\right)$-element identity matrix.

Assume that the components of the transmitted symbol vector $\mathbf{s}$ are obtained using the bit-to-symbol mapping function $s_{j}=\operatorname{map}\left(\mathbf{d}_{j}\right), j=1,2, \cdots, N_{T}$, where $\mathbf{d}_{j}=$ $\left[d_{j, 1}, d_{j, 2}, \cdots, d_{j, M_{c}}\right]^{T} \in\{+1,-1\}^{M_{c}}$ is the vector of data bits, and $M_{c}=\log _{2} M$ is the number of bits per $M$-QAM symbol. The vector of bits corresponding to $\mathbf{s}$ is denoted as $\mathbf{b}$, which satisfies $\mathbf{s}=\operatorname{map}(\mathbf{b})$ and is formed by concatenating the $N_{T}$ antennas' bits $\mathbf{d}_{1}, \mathbf{d}_{2}, \cdots, \mathbf{d}_{N_{T}}$, yielding $\mathbf{b}=\left[b_{1}, b_{2}, \cdots, b_{k}, \cdots, b_{M_{c} N_{T}}\right]=\left[\mathbf{d}_{1}^{T}, \mathbf{d}_{2}^{T}, \cdots, \mathbf{d}_{N_{T}}^{T}\right]^{T} \in$ $\{+1,-1\}^{M_{c} N_{T}}$. At the receiver, the task of the conventional symbol-based PDA MIMO detector is to seek a small BER or SER by estimating the a posteriori symbol probabilities $P\left(s_{j}=a_{m} \mid \mathbf{y}\right)=P_{m}\left(s_{j} \mid \mathbf{y}\right)$, without an exhaustive search in the space of all possible $M$-QAM MIMO symbol combinations. In this paper, our goal is to attain a high detection performance by estimating the a posteriori bit probabilities $P\left(d_{j, q}= \pm 1 \mid \mathbf{y}\right), q=1,2, \cdots, M_{c}$.

\section{UNIFIED MATRIX REPRESENTATION OF QAM}

The conventional description of the bit-to-symbol mapping process of QAM is based on a look-up table method [9]. To some extent, this method conceals the mathematical characteristic of a specific bit-to-symbol mapping process.

In MIMO systems, the mapping from bits to symbols may be compactly formulated by a unified transformation to be outlined below. Note that QAM signals are two-dimensional, i.e. we have $a_{m}=x+i y, \forall m \in\{1,2, \cdots, M\}$ where $x$ and $y$ are taken from the real alphabets $\mathcal{A}_{\text {real }}$ and $\mathcal{A}_{\text {imag }}$, respectively. For simplicity, we assume that each transmit antenna uses the same modulation scheme of $\mathcal{A}_{\text {real }}=\mathcal{A}_{\text {imag }}=$ $\overline{\mathcal{A}}=\left\{\alpha_{1}, \alpha_{2}, \cdots, \alpha_{\sqrt{M}}\right\}$ for square QAM, although our approach may be generalized to different alphabets for the real and imaginary parts. Let us now consider the bit-to-symbol mapping function $s_{j}=\operatorname{map}\left(\mathbf{d}_{j}\right)$ defined in Section II, and rewrite this function as

$s_{j}=s_{j}^{\Re}+i s_{j}^{\Im}=\operatorname{map}\left(d_{j, 1}^{\Re}, \cdots, d_{j, \frac{M_{c}}{2}}^{\Re}, d_{j, \frac{M_{c}}{2}+1}^{\Im}, \cdots, d_{j, M_{c}}^{\Im}\right)$

where $\Re$ and $\Im$ indicate real and imaginary parts of $s_{j}$, respectively, and $s_{j}^{\Re}, s_{j}^{\Im} \in \overline{\mathcal{A}}$. Since square QAM constellations having symmetric real and imaginary parts are considered here, it is sufficient to consider the real part only in the following derivation of the matrix representation without any loss of generality.

We assume that the elements of $\overline{\mathcal{A}}$ are placed in ascending order for constructing the vector $\mathbf{a}=\left[\alpha_{1}, \alpha_{2}, \cdots, \alpha_{\sqrt{M}}\right]^{T}$ and denote the corresponding bit strings as the $\left(\sqrt{M} \times \frac{M_{c}}{2}\right)$ element matrix $\mathbf{B}$, in which the $k$-th row is the bit string corresponding to $\alpha_{k}, k=1,2, \cdots, \sqrt{M}$. Then the bit-tosymbol mapping rule is described as

$$
\mathbf{B x}=\mathbf{a},
$$

where we would like to express $\mathbf{x}$, namely the "sub-generating unit", which maps the bits to the real part of a QAM symbol. Furthermore, $\mathbf{g}=\left[\mathbf{x}^{T}, i \mathbf{x}^{T}\right]$ is defined as the "generating unit" of $M$-QAM and it maps the bits to a QAM symbol.

\section{A. Linear Natural Bit-to-Symbol Mapping}

Let us take the linear natural mapping based 64QAM as an example. The alphabet is given by $\mathcal{A}_{64 \mathrm{QAM}}=$ $\{-7,-5,-3,-1,+1,+3,+5,+7\}, \quad M_{c}=6, \mathbf{B}=$ $[-1,-1,-1 ;-1,-1,+1 ;-1,+1,-1 ;-1,+1,+1 ;+1,-1,-1$; $+1,-1,+1 ;+1,+1,-1 ;+1,+1,+1$ ] (rows in $\mathbf{B}$ are distinguished by “;”), and $\mathbf{a}=[-7,-5,-3,-1,+1,+3,+5,+7]^{T}$, thus we have $\mathbf{x}=[4,2,1]^{T}$ in the light of Eq. (3). Similarly, based on Eq. (3), we have $\mathbf{x}=[2,1]^{T}$ for 16QAM, where $\mathbf{B}=[-1,-1 ;-1,+1 ;+1,-1 ;+1,+1]$, and $\mathbf{a}=[-3,-1,+1,+3]^{T}$. For 4QAM, $\mathbf{B}=[-1 ;+1]$, and $\mathbf{a}=[-1,+1]^{T}$, hence we have $\mathbf{x}=1$ according to Eq. (3). In comparison to the Gray mapping scenario, the Hamming distances between the adjacent constellation points of both 64QAM and 16QAM in the linear natural mapping case are either 1 or 2. For 4QAM however, the linear natural mapping presented here is identical to the Gray mapping.

From the above derivations provided for 4QAM, 16QAM and 64QAM, we can infer that for general rectangular QAM the modulation matrix is given by

$$
\mathbf{W}=\left(\begin{array}{ccc}
\mathbf{g} & \cdots & \mathbf{0} \\
\vdots & \ddots & \vdots \\
\mathbf{0} & \cdots & \mathbf{g}
\end{array}\right)_{N_{T} \times M_{c} N_{T}}
$$

where $\mathbf{g}=\left[2^{\frac{M_{c}}{2}-1}, 2^{\frac{M_{c}}{2}-2}, \cdots, 1,2^{\frac{M_{c}}{2}-1} i, 2^{\frac{M_{c}}{2}-2} i, \cdots, i\right]$ for square QAM. By contrast, for the rectangular QAM constellations conveying an odd number of bits, such as 8QAM, 32QAM etc., under the assumption that the real alphabet of the odd-throughput rectangular QAM is the same as that of its double-sized even-throughput QAM counterpart, we arrive at $\mathbf{g}=\left[2^{\frac{M_{c}-1}{2}}, 2^{\frac{M_{c}-1}{2}-1}, \cdots, 1,2^{\frac{M_{c}-1}{2}-1} i, \cdots, i\right]$. This statement is also valid for BPSK as a special case of a rectangular QAM signal with its imaginary part being 0 .

\section{B. Binary Reflected Gray Bit-to-Symbol Mapping}

In Section III, we presented a UMR of general rectangular QAM constellations using linear natural mapping for MIMO systems. However, since the nonlinear Gray mapping is typically regarded as the optimal labelling scheme ${ }^{2}$, two interest-

\footnotetext{
${ }^{2}$ Although there may be distinct Gray labellings that result in different biterror probability, especially in high-order constellations, the binary reflected Gray mapping has been shown to give the lowest possible average probability of bit errors for conventional symbol-based detection under certain assumptions on the channel [10]. We will simply use "Gray mapping" to refer to the "binary reflected Gray mapping" in the rest of the paper.
} 
TABLE I

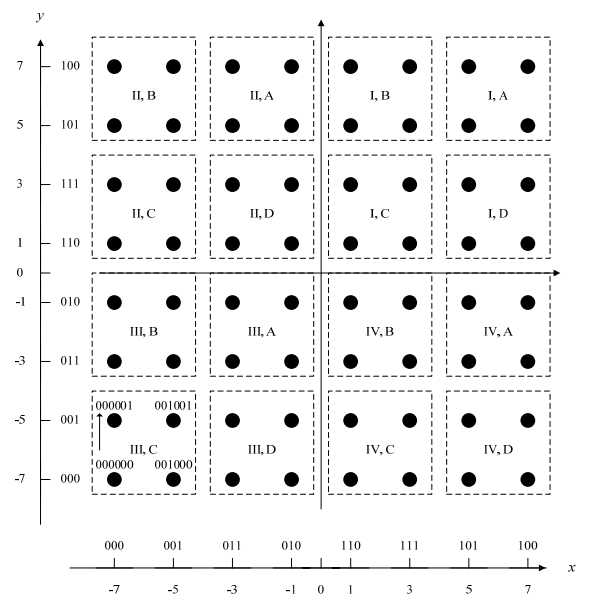

Fig. 1. Signal space diagram for 64QAM under Gray mapping

ing questions arise: 1) Would the linear natural mapping based B-PDA detector's performance degrade for high-order QAM owing to the lack of Gray mapping in the context of uncoded systems? 2) Is the Gray mapping still the best labelling scheme for the B-PDA MIMO detector? In order to answer these questions, in this section, we extend the previous linear natural mapping based results to the nonlinear Gray mapping case (for 4QAM, the linear natural and the Gray bit-to-symbol mappings are identical).

To elaborate further, let us consider rectangular 64QAM as an example. According to Eq. (3) and to the Gray mapping rule shown in Fig. 1, we have $\mathbf{a}=[-7,-5,-3,-1,+1,+3,+5$, $+7]^{T}, \mathbf{x}=[a, b, c]^{T}$, and the original Gray mapping based bits $000,001,011,010,110,111,101,100$ are converted into the bipolar representation of $\mathbf{B}=[-1,-1,-1 ;-1,-1,+1 ;-1$, $+1,+1 ;-1,+1,-1 ;+1,+1,-1 ;+1,+1,+1 ;+1,-1,+1 ;+1$, $-1,-1]$ under the rule of $2 b_{i}-1$, where $b_{i}, i=1,2$, is a binary digit of 0 or 1 . Substituting $\mathbf{a}, \mathbf{B}$ and $\mathbf{x}$ into Eq. (3), owing to the non-linearity of Gray mapping, $\mathbf{x}$ does not have a unique solution. However, each of the following four sub-systems of equations has a unique solution:

$$
\begin{gathered}
\text { i) }\left\{\begin{array}{l}
-a-b-c=-7 \\
-a-b+c=-5
\end{array} \Rightarrow(a, b, c)=(4,2,1)\right. \\
i i)\left\{\begin{array}{l}
-a+b+c=-3 \\
-a+b-c=-1
\end{array} \Rightarrow(a, b, c)=(4,2,-1)\right. \\
\{i i i)\left\{\begin{array}{l}
a+b-c=1 \\
a+b+c=3
\end{array} \Rightarrow(a, b, c)=(4,-2,1)\right. \\
i v)\left\{\begin{array}{l}
a-b+c=5 \\
a-b-c=7
\end{array} \Rightarrow(a, b, c)=(4,-2,-1)\right.
\end{gathered}
$$

Observe in Eq. (5) and Eq. (6) that there are four possible solution vectors acting as the sub-generating unit, namely $\mathbf{x}_{1}=[4,2,1]^{T}, \mathbf{x}_{2}=[4,2,-1]^{T}, \mathbf{x}_{3}=[4,-2,1]^{T}$ and $\mathbf{x}_{4}=[4,-2,-1]^{T}$ corresponding to four 3-bit tuples (every three adjacent bits as a unit), in which $(-1,-1),(-1,1)$, $(1,1)$ and $(1,-1)$ are the first two bits, respectively. For example, if the 3-bit tuple to be transmitted is $(-1,-1, x)$, then its corresponding sub-generating unit is $\mathbf{x}_{1}$, and if the

\begin{tabular}{|c|c|}
\hline 16QAM $\left(M_{c}=4\right)$ & 64QAM $\left(M_{c}=6\right)$ \\
\hline $\begin{array}{l}\text { for } k=1: \frac{M_{C}}{2}: M_{c} N_{T} \\
\text { for } j=1: N_{T} \\
\text { if } b_{k}==1 \\
\quad \mathbf{W}(j, k+1)=-\mathbf{W}(j, k+1) \\
\text { end } \\
\text { end } \\
\text { end }\end{array}$ & $\begin{array}{l}\text { for } k=1: \frac{M_{c}}{2}: M_{c} N_{T} \\
\text { for } j=1: N_{T} \\
\text { if } b_{k}==1 \& \& b_{k+1}==1 \\
\mathbf{W}(j, k+1)=-\mathbf{W}(j, k+1) \\
\text { elseif } b_{k}==-1 \& \& b_{k+1}==1 \\
\mathbf{W}(j, k+2)=-\mathbf{W}(j, k+2) \\
\text { elseif } b_{k}=1 \& \& b_{k+1}==-1 \\
\mathbf{W}(j, k+1)=-\mathbf{W}(j, k+1) \\
\quad \mathbf{W}(j, k+2)=-\mathbf{W}(j, k+2) \\
\text { end } \\
\text { end } \\
\text { end }\end{array}$ \\
\hline
\end{tabular}

UMR RULE OF GRAY MAPPING

3-bit tuple is $(1,-1, x)$, then we have $\mathbf{x}_{4}$, where $x$ is -1 or 1.

By jointly considering Fig. 1 and the four sub-generating units $\mathbf{x}_{1}, \cdots, \mathbf{x}_{4}$, we may infer several pieces of useful information for 64QAM. Firstly, the component having the largest modulus in the generating unit remains the same as that of its counterpart in linear natural mapping, but the signs of the smaller components may change. Secondly, the set of constellation points which dwell in the same four-point-block within the dashed box of Fig. 1 share the same generating unit, Consequently, 64QAM is divided into 16 four-point-blocks and has 16 different generating units constructed by 4 different sub-generating units. Furthermore, the constellation points in the same half plane are described by Eq. (5) (the left half plane) and Eq. (6) (the right half plane), each of which is composed by two further sets of equations, as seen in i), ii), iii) and iv) of Eq. (5) and Eq. (6), respectively. In addition, it is plausible that the solution of $(a, b, c)$ for each of i), ii), iii) and iv) is unique under the constraint that i) and ii) constituting the left half plane share the same values of $(a, b)$, and so do iii) and iv) constituting the right half plane.

Based on the above insights drawn for 64QAM, the UMR of the most commonly used Gray mapping may be obtained by appropriately alternating the sign of certain entries in the above static UMR of the linear natural mapping based QAM, which in fact serves as the basis matrix for Gray mapping aided QAM and may be prestored for access. The procedures of selecting the appropriate generating unit is illuminated using 16QAM and 64QAM as our examples in Table I. For ease of exposition, Matlab-style pseudo-code is used.

\section{B-PDA MIMO DETECTION BASED ON UMR}

\section{A. Basic Detection Algorithm}

Based on the UMR of QAM, we have $\mathbf{s}=\mathbf{W b}$ and $\mathbf{s}=$ $\mathbf{W}(\mathbf{b}) \mathbf{b}$ for the linear natural mapping and nonlinear Gray mapping, respectively. For ease of exposition, we will consider the linear mapping based rectangular QAM as an example for elaborating on the B-PDA detector. Thus the original system model of (1) may be rewritten as

$$
\mathbf{y}=\mathbf{H W} \mathbf{b}+\mathbf{n}=\mathbf{Q b}+\mathbf{n},
$$

where $\mathbf{Q}=\mathbf{H W}$ captures the combined effect of both the channel matrix and of the bit-to-symbol mapping matrix. We can see from Eq. (7) that the original QAM detection problem has been transformed to a BPSK-like detection model.

Adopting the non-decorrelated signal model of [5], Eq. (7) can be further re-formulated as

$$
\mathbf{y}=\mathbf{q}_{l} b_{l}+\sum_{k \neq l} \mathbf{q}_{k} b_{k}+\mathbf{n} \triangleq \mathbf{q}_{l} b_{l}+\mathbf{v}_{l}
$$


where $\mathbf{q}_{l}$ denotes the $l$-th column of $\mathbf{Q}$, and $b_{l}$ is the $l$-th bit of $\mathbf{b}$, while $\mathbf{v}_{l}$ is the interference and noise term contaminating bit $b_{l}, l, k=1,2, \cdots, M_{c} N_{T}$.

For each bit $b_{l}$, we define an $M$-element bit probability vector $\mathbf{P}(l)$ whose $m$-th element $P_{m}\left(b_{l} \mid \mathbf{y}\right)$ is the current estimate of the a posteriori probability that $b_{l}=a_{m}$, where $m=1,2$ and $a_{1}=+1, a_{2}=-1$. The key idea of the PDA algorithm [2] is to approximate the interference plus noise term $\mathbf{v}_{l}$ as an $N_{R}$-variate colored Gaussian distributed random variable with a mean of $\mu_{l}=\sum_{k \neq l} \bar{b}_{k} \mathbf{q}_{k}$, covariance of $\mathbf{V}_{l}=\sum_{k \neq l} \mathbf{V}_{b_{k}} \mathbf{q}_{k} \mathbf{q}_{k}^{H}+N_{0} \mathbf{I}$, and pseudo-covariance of $\mathbf{U}_{l}=\sum_{k \neq l} \mathbf{U}_{b_{k}} \mathbf{q}_{k} \mathbf{q}_{k}^{T}$, where $\bar{b}_{k}=\sum_{m=1}^{2} a_{m} P_{m}\left(b_{k} \mid \mathbf{y}\right)$, $\mathbf{V}_{b_{k}}=\sum_{m=1}^{2}\left(a_{m}-\bar{b}_{k}\right)\left(a_{m}-\bar{b}_{k}\right)^{*} P_{m}\left(b_{k} \mid \mathbf{y}\right)$, and $\mathbf{U}_{b_{k}}=$ $\sum_{m=1}^{2}\left(a_{m}-\bar{b}_{k}\right)\left(a_{m}-\bar{b}_{k}\right)^{T} P_{m}\left(b_{k} \mid \mathbf{y}\right)$.

Here $P_{m}\left(b_{k} \mid \mathbf{y}\right)$ is initialized as a uniform distribution and will be replaced with an updated bit-probability at each iteration of the B-PDA detector.

$$
\begin{aligned}
& \text { Let } \mathbf{w}=\mathbf{y}-b_{l} \mathbf{q}_{l}-\sum_{k \neq l} \bar{b}_{k} \mathbf{q}_{k} \text { and } \\
& \varphi_{m}\left(b_{l}\right) \triangleq \exp \left(-\left(\begin{array}{c}
\Re(\mathbf{w}) \\
\Im(\mathbf{w})
\end{array}\right)^{T} \boldsymbol{\Lambda}_{l}\left(\begin{array}{c}
\Re(\mathbf{w}) \\
\Im(\mathbf{w})
\end{array}\right)\right),
\end{aligned}
$$

where we have

$$
\mathbf{\Lambda}_{l} \triangleq\left(\begin{array}{cc}
\Re\left(\mathbf{V}_{l}+\mathbf{U}_{l}\right) & -\Im\left(\mathbf{V}_{l}-\mathbf{U}_{l}\right) \\
\Im\left(\mathbf{V}_{l}+\mathbf{U}_{l}\right) & \Re\left(\mathbf{V}_{l}-\mathbf{U}_{l}\right)
\end{array}\right)^{-1}
$$

while $\Re(\cdot)$ and $\Im(\cdot)$ represent the real and imaginary part of a complex variable, respectively.

Since it is assumed that all the transmitted bits have equal a priori probabilities, the a posteriori bit probability is given as

$$
P_{m}\left(b_{l} \mid \mathbf{y}\right)=\frac{p_{m}\left(\mathbf{y} \mid b_{l}\right) P\left(b_{l}=a_{m}\right)}{\sum_{m=1}^{2} p_{m}\left(\mathbf{y} \mid b_{l}\right) P\left(b_{l}=a_{m}\right)} \approx \frac{\varphi_{m}\left(b_{l}\right)}{\sum_{m=1}^{2} \varphi_{m}\left(b_{l}\right)} .
$$

In summary, the algorithm proceeds as follows.

1) Initialization: set the initial values of the bit probabilities $P_{m}\left(b_{l} \mid \mathbf{y}\right)$ using a uniform distribution for $\forall l=$ $1,2, \cdots, M_{c} N_{T}, \forall m=1,2$, i.e. $P_{m}\left(b_{l} \mid \mathbf{y}\right)=0.5$; set the iteration counter to $z=1$.

2) Set the bit index to $l=1$.

3) Based on the current values of $\{\mathbf{P}(k)\}_{k \neq l}$, compute $P_{m}\left(b_{l} \mid \mathbf{y}\right)$ using (9) - (11), which will replace the value of the corresponding element of $\mathbf{P}(l)$.

4) If $l<M_{c} N_{T}$, let $l=l+1$ and go to step 3). Otherwise, go to step 5).

5) If $\mathbf{P}(l)$ has converged, $\forall l$, or the iteration index has reached its maximum, go to step 6). Otherwise, let $z=z+1$ and return to step 2).

6) For $l=1,2, \cdots, M_{c} N_{T}$, make a decision concerning $\hat{b}_{l}$ using $\hat{b}_{l}=a_{d}, d=\arg \max _{m^{\prime}=1,2}\left\{P_{m^{\prime}}\left(b_{l} \mid \mathbf{y}\right)\right\}$, yielding $\hat{\mathbf{b}}=$ $\left\{\hat{b}_{l} \mid l=1,2, \cdots, M_{c} N_{T}\right\}$.
TABLE II

PROBABILITIES COMPUTED IN ONE ITERATION FOR CPDA

\begin{tabular}{c|cccccc}
\hline & 1 & 2 & $\cdots$ & $m$ & $\cdots$ & $M$ \\
\hline $\mathbf{P}(1)$ & $P_{1}\left(s_{1} \mid \mathbf{y}\right)$ & $P_{2}\left(s_{1} \mid \mathbf{y}\right)$ & $\cdots$ & $P_{m}\left(s_{1} \mid \mathbf{y}\right)$ & $\cdots$ & $P_{M}\left(s_{1} \mid \mathbf{y}\right)$ \\
$\mathbf{P}(2)$ & $P_{1}\left(s_{2} \mid \mathbf{y}\right)$ & $P_{2}\left(s_{2} \mid \mathbf{y}\right)$ & $\cdots$ & $P_{m}\left(s_{2} \mid \mathbf{y}\right)$ & $\cdots$ & $P_{M}\left(s_{2} \mid \mathbf{y}\right)$ \\
$\vdots$ & $\vdots$ & $\vdots$ & $\ldots$ & $\vdots$ & $\cdots$ & $\vdots$ \\
$\mathbf{P}(j)$ & $P_{1}\left(s_{j} \mid \mathbf{y}\right)$ & $P_{2}\left(s_{j} \mid \mathbf{y}\right)$ & $\cdots$ & $P_{m}\left(s_{j} \mid \mathbf{y}\right)$ & $\cdots$ & $P_{M}\left(s_{j} \mid \mathbf{y}\right)$ \\
$\vdots$ & $\vdots$ & $\vdots$ & $\ldots$ & $\vdots$ & $\cdots$ & $\vdots$ \\
$\mathbf{P}\left(N_{T}\right)$ & $P_{1}\left(s_{N_{T}} \mid \mathbf{y}\right)$ & $P_{2}\left(s_{N_{T}} \mid \mathbf{y}\right)$ & $\cdots$ & $P_{m}\left(s_{N_{T}} \mid \mathbf{y}\right)$ & $\cdots$ & $P_{M}\left(s_{N_{T}} \mid \mathbf{y}\right)$ \\
\hline
\end{tabular}

\section{B. Discussions}

From the above procedures we can see that the size of the detected vector is extended from $N_{T}$ symbols to $M_{c} N_{T}$ bits and the number of constellation points is reduced from $M \geq 4$ to 2. It was demonstrated in [5] that the accuracy of "Gaussian approximation" and therefore that of the soft outputs is the best for a large number of transmit antennas and a small number of constellation points. In this context the question arises as to how will the UMR of QAM influence the achievable performance of the proposed B-PDA? This may not be an easy dilemma to resolve, since the theoretical performance bound of PDA based algorithms remains an open problem, and the UMR transformation may have both a positive and a negative impact on the achievable performance. Firstly, based on the observations in [5], we intuitively infer that our approach may be interpreted as though it virtually increased the number of transmit antennas, while reducing the QAM constellation to a binary constellation. This approach improves the accuracy of the Gaussian approximation and, additionally, it reduces the decision ambiguity concerning the symbols of high-order QAM, since it leads to binary decisions. Hence the performance of B-PDA is expected to be enhanced. On the other hand, the UMR transformation may result in a degraded composite channel of $\mathbf{Q}$, which may deteriorate the achievable performance of B-PDA. In fact, the composite effect of the UMR is positive, as it will be evidenced in Section VI.

Furthermore, the design and choice of constellation labelling may have a significant impact on the BER performance of symbol-based detectors. This explains why the Gray mapping is generally regarded as the optimal labelling scheme. However, the specific bit assignment within a symbol does not affect the SER performance, because the SER performance is determined by the minimum Euclidean distance of the constellation employed. Since the proposed B-PDA skips the symbol-detection stage and directly operates at the bit-level, where the bits are treated separately, the correlation between bits may not affect the achievable BER performance, as seen from the simulation results in Section VI.

\section{Complexity Analysis}

It was shown in [6] that the conventional CPDA MIMO detector has to update an $\left(M \times N_{T}\right)$-element probabilitymatrix at each iteration, until all the entries in the matrix converge or the maximum iteration index is reached. For the sake of compactness, these probabilities are given in Table II. Consequently, the number of probabilities to be computed may be as high as $M N_{T} N_{i t}$ for each symbol vector s, where $N_{i t}$ is the average number of iterations required for convergence in the process of detecting each $M$-QAM $N_{T}$-symbol vector. 
By comparison, it may be readily observed from the above B-PDA procedures that provided the UMR of QAM is employed, the corresponding probability-matrix for B-PDA has $M_{c} N_{T}$ rows and two columns, thus the number of probabilities to be computed reduces to $2 M_{c} N_{T} N_{i t}^{\prime}$, where $N_{i t}^{\prime}$ is the average number of iterations required for detecting each corresponding bit vector. Additionally, the complexity imposed by computing a single probability is denoted as $C_{p}$ and $C_{p}^{\prime}$ for CPDA and B-PDA, respectively. Then, the achievable complexity ratio of the B-PDA MIMO detector over the conventional PDA MIMO detector required for decoding a single symbol vector becomes

$$
R_{c}(M)=\frac{2 M_{c} N_{T} N_{i t}^{\prime} C_{p}^{\prime}}{M N_{T} N_{i t} C_{p}}=\frac{2 \log _{2}(M)}{M} \cdot \frac{N_{i t}^{\prime}}{N_{i t}} \cdot \frac{C_{p}^{\prime}(M)}{C_{p}(M)},
$$

where $2 \log _{2}(M) C_{p}^{\prime}(M)$ and $M C_{p}(M)$ are the per-iterationcomplexity for B-PDA and CPDA, respectively, while $2 \log _{2}(M) N_{i t}^{\prime} C_{p}^{\prime}(M)$ and $M N_{i t} C_{p}(M)$ are the complexity per symbol vector for B-PDA and CPDA, respectively. Eq. (12) is a monotonically decreasing function of the modulation order $M$, because the ratio $\frac{N_{i t}^{\prime}}{N_{i t}}$ of the number of iterations is typically close to 1 , while $\frac{C_{p}^{\prime}(M)}{C_{p}(M)}$ is less than 1 and decreases upon increasing $M$, as it will be demonstrated in Fig. $2^{3}$. Consequently, we have $R_{c}(M) \ll 1$ for $M \gg 4$. We provide a demonstrative example in Fig. 2 for the complexity comparison between the conventional symbol-based CPDA and the proposed B-PDA quantified in terms of the number of FLoating point OPerations (FLOPs) per iteration and per symbol vector for different QAM constellations. It may be readily seen that the complexity of B-PDA using the linear natural bit mapping is reduced by about $80 \%$ for the system configuration of 64QAM, $N_{T}=N_{R}=2, \mathrm{SNR}=20 \mathrm{~dB}$.

\section{Simulation Results}

In this section, we characterize the achievable performance of the B-PDA algorithm using Monte Carlo simulations in the context of the VBLAST system [11] as a function of the average SNR per receive antenna (SNR $\triangleq$ $10 \log _{10}\left(E\left\{\|\mathbf{H s}\|^{2} / N_{R}\right\} / N_{0}\right)$ ) for transmission over flat Rayleigh fading channels, where the entries of the MIMO channel are independent and identically distributed (i.i.d.), zero mean, unit-variance complex-valued Gaussian random variables. A new independent channel realization is drawn for each transmitted symbol vector. The noise vector $\mathbf{n}$ is i.i.d. $\mathcal{C N}\left(0, N_{0}\right)$. No optimal ordering of the bits is performed before detection in the following PDA related simulations for the sake of fair comparison with the results of [6]. The convergence threshold is set to $\varepsilon=\left|P_{i, j}^{z+1}-P_{i, j}^{z}\right|=0.001$, where $P_{i, j}^{z}$ represents the $(i, j)$-th value of the probabilitymatrix of CPDA or B-PDA at the $z$-th iteration.

In order to choose the appropriate number of iterations for the PDA-aided MIMO detectors, Fig. 3 evaluates the impact of the number of iterations both on the performance of the conventional symbol-based CPDA of [6] using Gray mapping based 16QAM and on the proposed B-PDA using linear natural mapping based 16QAM in the context of $(2 \times 2)$-antenna aided

\footnotetext{
${ }^{3}$ The average number of iterations of the conventional PDA MIMO detector is typically $3-5$ as seen in both [2][4] as well as in Fig. 2 and Fig. 3. In addition, the computational cost expressed in terms of FLOPs for estimating the a posteriori probability of a BPSK-like signal is lower than that of a complex-valued multilevel QAM signal.
}

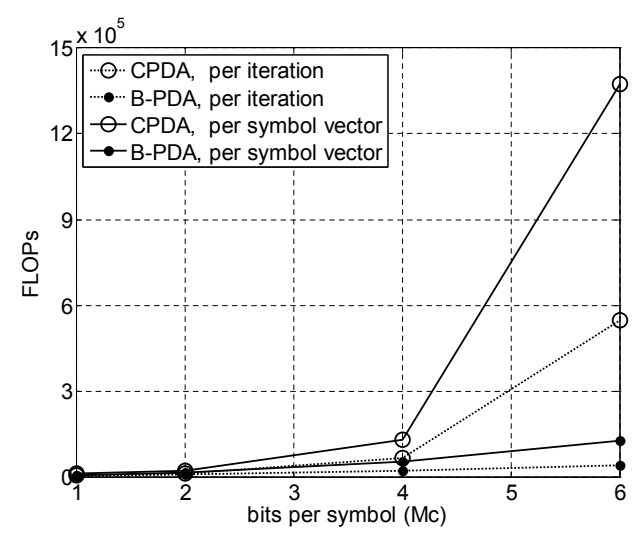

Fig. 2. Complexity comparison in FLOPs per iteration and per symbol vector for B-PDA with linear natural bit mapping and CPDA with Gray bit mapping using BPSK $\left(M_{c}=1\right)$, 4QAM $\left(M_{c}=2\right)$, 16QAM $\left(M_{c}=4\right)$, and 64QAM $\left(M_{c}=6\right), \mathrm{SNR}=20 \mathrm{~dB}, N_{T}=N_{R}=2$

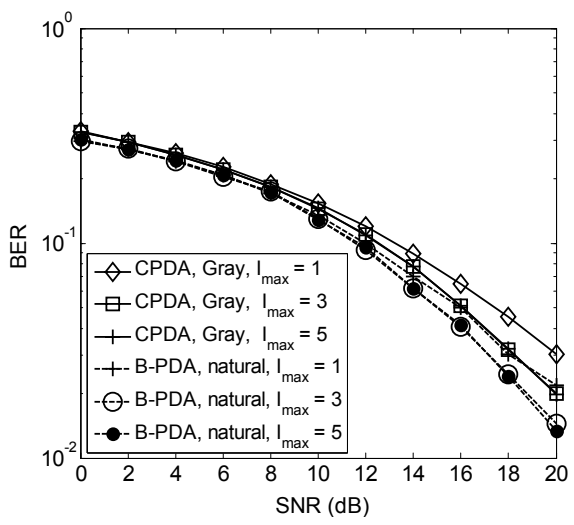

Fig. 3. Impact of the number of iterations on the achievable performance of CPDA and B-PDA in VBLAST using 16QAM, $N_{T}=N_{R}=2$

VBLAST systems. The maximum number of iterations $I_{\max }$ is set to 1,3 , and 5, respectively. It can be seen from Fig. 3 that both the CPDA and B-PDA exhibit quite a good convergence, since the performance loss is modest even when the maximum number of iterations is set to be as low as $I_{\max }=3$.

Based on the above observation, we set the maximum number of iterations to $I_{\max }=5$ in the following investigations, where B-PDA is compared to the conventional symbol-based CPDA, MMSE-Ordered-SIC (MMSE-OSIC) and ML (it is implemented using the more efficient Sphere Decoder of [12]), while employing both the linear natural bit mapping and the nonlinear Gray mapping ${ }^{4}$. Fig. 4 and Fig. 5 evaluate the attainable BER and SER performance of 64QAM, respectively. 5 . In general, the performance of B-PDA is superior to that of CPDA, and the attainable gain is more substantial in terms

\footnotetext{
${ }^{4}$ When Gray mapping based rectangular QAM is used, a realistic detector should be used to first estimate the $\mathbf{W}(\mathbf{b})$ at the receiver. Apart from the additional complexity, there would be a performance degradation associated with the quality of the corresponding estimation algorithm. However, in order to provide a lower bound performance for the linear natural mapping based B-PDA, we assume that the modulation matrix $\mathbf{W}(\mathbf{b})$ of the Gray mapping scenario is perfectly known at the receiver.

${ }^{5}$ For high-order modulation, multiple bit errors occurring close to each other will probably create only one symbol error. In this situation, the SER is a useful metric, which is widely adopted in the literature regarding high-order $M$-ary signaling detection in MIMO systems, as seen in [4] and [7].
} 


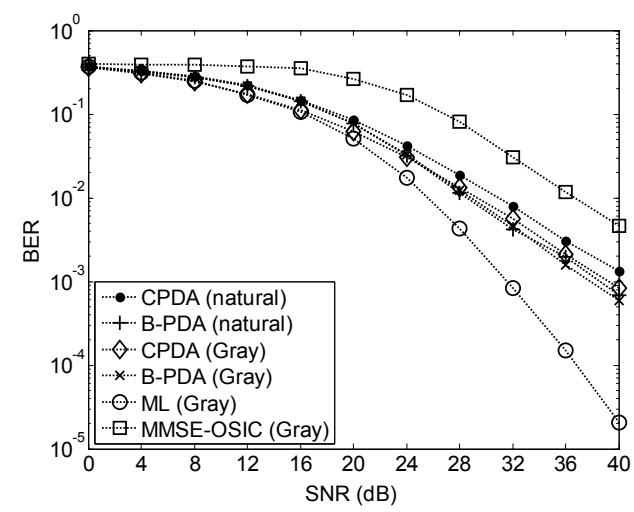

Fig. 4. BER Comparison of B-PDA and CPDA, MMSE-OSIC, ML in VBLAST with 64QAM, $I_{\max }=5, N_{T}=N_{R}=2$

of SER. For the linear natural mapping based 64QAM, BPDA outperforms CPDA by approximately $3 \mathrm{~dB}$ at both $\mathrm{BER}=10^{-2}$ and $\mathrm{SER}=10^{-2}$. When the conventional symbol-based CPDA uses the nonlinear Gray mapping, it can be seen by comparing the results of Fig. 4 to those of Fig. 5 that the BER performance of the linear natural mapping B-PDA slightly erodes at low SNRs, but it still has an edge over that of CPDA at high SNRs. Furthermore, observe from the SER curves of Fig. 5 that the SNR gain of the linear natural mapping based B-PDA remains superior to that of CPDA at all the SNR values considered. This is because Gray mapping is efficient in terms of reducing the BER as a benefit of the binary labelling gain, but it is unable to improve the SER, since the SER is determined by the minimum Euclidean distance of the constellation points (a single constellation may have various labelling schemes). It is also interesting to observe that the performance of the linear natural mapping aided QAM approaches that of the Gray mapping aided QAM for the proposed B-PDA, while the latter represents a lower bound performance valid under the assumption of perfect knowledge of $\mathbf{W}$ at the receiver. In other words, B-PDA is insensitive to the specific mapping rule, as long as the matrix $W$ is known at the receiver. This is because it operates directly on the basis of the separate bits, namely in a bit-by-bit fashion which treats each bit independently and remains unaffected by the specific correlation between the bits within a symbol. By comparison, the conventional PDA generally makes the decision at the symbol-level using the estimated a posteriori symbol-probabilities, and if necessary, the bits are recovered according to the inverse bit-to-symbol mapping, which retains the better BER of Gray mapping but has no SER advantage. Therefore, it may be concluded that it is not necessary to design complex bit-to-symbol mapping rules for the proposed B-PDA, because it is capable of approaching the lower bound performance provided by the Gray mapping based B-PDA in ideal conditions even upon using the simplest linear natural bit-to-symbol mapping.

\section{CONCLUSIONS}

We proposed a unified B-PDA detection scheme for spatial multiplexing MIMO systems. Based on the UMR of high-order rectangular QAM, the B-PDA transforms the symbol detection process of QAM to that of a BPSK-like scenario with the aid of a composite channel matrix combining the effect of

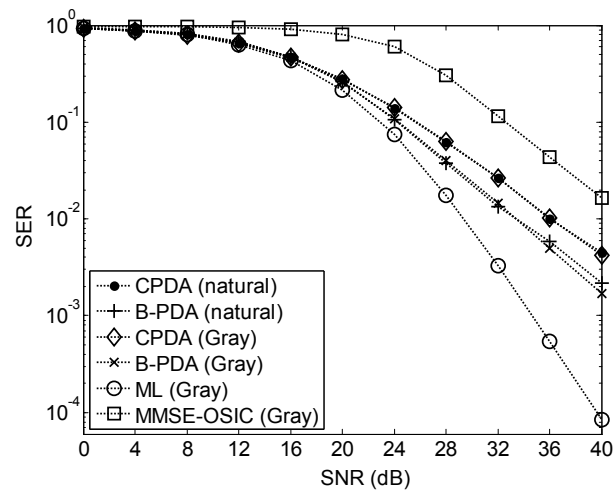

Fig. 5. SER Comparison of B-PDA and CPDA, MMSE-OSIC, ML in VBLAST with 64QAM, $I_{\max }=5, N_{T}=N_{R}=2$

modulation and of the original channel matrix. Simulations and complexity analysis demonstrate that the proposed B-PDA outperforms the conventional CPDA, despite dramatically reducing complexity, especially in the context of high-order QAM constellations. Additionally, the simulation results show that the linear natural mapping based B-PDA is insensitive to the choice of labelling scheme and approaches the lower bound performance provided by the Gray mapping based BPDA under the perfect modulation matrix assumption. Finally, we concluded that it is preferable to use the simpler and more practicable linear natural bit-to-symbol mapping rather than Gray mapping for the B-PDA aided MIMO detector in the uncoded VBLAST-style systems considered.

\section{REFERENCES}

[1] Y. Bar-Shalon and X. R. Li, Estimation and Tracking: Principles, Techniques and Software. Dedham, MA: Artech House, 1993.

[2] J. Luo, K. R. Pattipati, P. K. Willett, and F. Hasegawa, "Near optimal multiuser detection in synchronous CDMA using probabilistic data association," IEEE Communications Letters, vol. 5, no. 9, pp. 361-363, Sept. 2001.

[3] D. Pham, J. Luo, K. Pattipati, and P. Willett, "A PDA-Kalman approach to multiuser detection in asynchronous CDMA," IEEE Communications Letters, vol. 6, no. 11, pp. 475-477, Nov. 2002.

[4] D. Pham, K. Pattipati, P. Willet, and J. Luo, "A generalized probabilistic data association detector for multiple antenna systems," IEEE Communications Letters, vol. 8, no. 4, pp. 205-207, Apr. 2004.

[5] J. Fricke, M. Sandell, J. Mietzner and P. Hoeher, "Impact of the Gaussian approximation on the performance of the probabilistic data association MIMO decoder," EURASIP Journal on Wireless Communications and Networking, vol. 5, no. 5, pp. 796-800, Oct. 2005.

[6] Y. Jia, C. M. Vithanage, C. Andrieu and R. J. Piechocki, "Probabilistic data association for symbol detection in MIMO systems," Electronics Letters, vol. 42, no. 1, pp. 38-40, Jan. 2006.

[7] Z. Mao, X. Wang and X. Wang, "Semidefinite programming relaxation approach for multiuser detection of QAM signals" IEEE Transactions on Wireless Communications, vol. 6, no. 12, pp. 4275-4279, Dec. 2007.

[8] T. Matsumoto, S. Ibi, S. Sampei, and R. Thoma, "Adaptive transmission with single-carrier multilevel BICM," Proceedings of the IEEE, vol. 95, no. 12 , pp. 2354-2367, Dec. 2007.

[9] L. Hanzo, S. X. Ng, T. Keller and W. Webb, Quadrature Amplitude Modulation: From Basics to Adaptive Trellis-Coded, Turbo-Equalised and Space-Time Coded OFDM, CDMA and MC-CDMA Systems, 2nd edition. Wiley-IEEE Press, 2004.

[10] E. Agrell, J. Lassing, E. Ström, and T. Ottosson, "On the optimality of the binary reflected Gray code," IEEE Transactions on Information Theory, vol. 50, no. 12, pp. 3170-3182, Dec. 2004.

[11] P. W. Wolniansky, G. J. Foschini, G. D. Golden, and R. A. Valenzuela, "V-BLAST: an architecture for realizing very high data rates over the richscattering wireless channel," in Proceeding of IEEE URSI International Symposium on Signals, Systems, and Electronics, 1998, pp. 295-300, Italy, Sept. 1998.

[12] E. Viterbo and J. Boutros, "A universal lattice code decoder for fading channels," IEEE Transactions on Information Theory, vol. 45, no. 7, pp. 1639-1642, Jul. 1999. 